\title{
THE ANALYSIS OF THE EPR SPECTRA IN ZnO: Mn NANOCRYSTALS USING THE DERIVATIVE SPECTROSCOPY METHOD
}

\author{
O.V. Kovalenko, Ye.G. Plakhtii, O.V. Khmelenko, V.Yu. Vorovsky \\ Oles Honchar Dnipro National University, Dnipro, Ukraine \\ e-mail kovalenko.dnu@gmail.com
}

\begin{abstract}
A method for computer processing of experimental EPR spectra using the derivative spectroscopy method is proposed. Application of the proposed method to a wide, structureless line of the EPR spectrum in ZnO: Mn nanocrystals obtained by the cryochemical method made it possible to isolate the components of the spectrum, associate them with $\mathrm{Mn}^{2+}$ ions. Application of the proposed method to the analysis of the EPR spectrum ZnO: Mn nanocrystal's obtained by ultrasonic pyrolysis of an aerosol with a different activator concentration after annealing made it possible to reveal the presence of an hyperfine structure of the EPR spectrum of $\mathrm{Mn}^{2+}$ ions, which are located in different local environments. The constants of the detected spectra are determined.

Keywords EPR, ZnO: Mn nanocrystals, derivative spectroscopy method, hyperfine structure of the EPR spectrum, EPR spectrum constants.
\end{abstract}

Received 01.11.2019; Received in revised form 08.12.2019; Accepted 23.12.2019

\section{Introduction}

EPR is an effective method of non-destructive testing, widely used in chemistry, physics, biology, medicine [1-4]. It allows one to determine: the concentration of paramagnetic centers, the type of paramagnetic center responsible for the EPR signal, its valency, local symmetry of the crystal field in which the paramagnetic center is located, to study the kinetics of phase transitions, to analyze the recharge of paramagnetic centers due to external influences on the sample, etc. .

Further progress in the application of this method is associated, on the one hand, with the improvement of the hardware, which allows directly improving the resolution of the method [5-6]. On the other hand, a significant role in the effective analysis of experimental data is played by the methods of analysis of primary information [7-8]. In this regard, two problems must be overcome. The first problem is spin-spin interaction with an increase in the concentration of paramagnetic centers causes to a substantial broadening of the EPR lines, which, in turn, leads to a decrease in the informational significance of the obtained experimental data $[9,10]$. The second problem - due to the nonequivalence of the positions of paramagnetic centers, their EPR spectra, overlapping each other, complicate the analysis of experimental data $[11,12]$. This work proposes to use the derivative spectroscopy method (DSM) to analyze the primary experimental EPR spectra in order to determine the spectrum parameters. This method has already demonstrated its capabilities in the analysis of luminescence and absorption spectra in order to identify individual bands in the composition of wide lines [13]. Thanks to computer processing of the primary experimental spectra, the possibility of detecting the signals constituting the EPR spectrum, which is one wide absorption line, has been demonstrated. DSM made it possible to establish the components of the EPR spectra in a complicated signal, which is a superposition of two EPR spectra.

\section{Materials and methods}

DSM is used to analyze the smoothed EPR spectra according to the technique presented in [13]. Smoothing was performed by the Tikhonov method according to the technique described in [14]. As is known, from a mathematical point of view, the first derivative at the points of intersection of the spectrum with a zero value of the function gives us information about the location of the extrema in the initial spectrum. Odd derivatives provide us with the same information from the point of view of the DSM. Accordingly, obtaining odd derivatives 
of a higher order, we can obtain information on the components of the complicated EPR spectrum. It is necessary to take into account the fact, that the higher the order of the derivative, the greater the contribution of the noise signal to the result. Given the above, in the analysis it is necessary to reduce the order of derivatives to the third and fifth order of the "zero" spectrum, or second and fourth order of the initial EPR spectrum. The foregoing is due to the fact that the initial EPR spectrum itself is the first derivative of the absorption line. Accordingly, in this work, the maximum order of the derivative was the fourth order of the initial EPR spectrum.

$\mathrm{ZnO}$ : $\mathrm{Mn}$ nanocrystals (NC) were chosen as the object of study, which were synthesized by cryochemical synthesis with a concentration of $\mathrm{Mn}-4$ at.\% [15], as well as by the method of ultrasonic pyrolysis of aerosol with a concentration of $\mathrm{Mn}-2$ at.\% [16]. Acetates acted as precursors in the first case, and zinc and manganese nitrates in the other. The initial EPR spectra of such NC were recorded using a Radiopan SE / X-2543 radio spectrometer and consisted of 4096 points. At cryochemical synthesis, the EPR spectrum of the sample is a wide absorption line (Fig. 1a), which is apparently a superposition of narrower lines. This broad line is due to the action of hydrogen on the crystal structure of $\mathrm{ZnO} \mathrm{NC}$. Hydrogen, which is the product of the thermal decomposition of acetates, creates a large number of point defects during the formation of $\mathrm{ZnO}$ NC. Such defects contribute to the activation of the process of doping $\mathrm{ZnO} \mathrm{NC}$ with $\mathrm{Mn}^{2+}$ ions. It can be assumed that the structure of the observed EPR spectrum contains lines of the hyperfine structure (HFS) of $\mathrm{Mn}^{2+}$ ions, which isovalently replace $\mathrm{Zn}^{2+}$ ions at the crystal lattice sites of ZnO NC. Using the DSM, one can identify these lines and set the spectrum parameters. The EPR spectrum of $\mathrm{ZnO} \mathrm{NC}$ obtained by ultrasonic pyrolysis of aerosol after annealing at a temperature of $\mathrm{T}=550{ }^{\circ} \mathrm{C}$ probably represents a superposition of two EPR spectra of $\mathrm{Mn}^{2+}$ ions located at the crystal lattice sites of $\mathrm{ZnO}$ $\mathrm{NC}$ and on the surface of $\mathrm{ZnO}$ : Mn NC (Fig.2a). $\mathrm{Mn}^{2+}$ ions located on the surface of NC are in a different local environment compared to those $\mathrm{Mn}^{2+}$ ions that have introduced into the sites of the crystal lattice of $\mathrm{ZnO} \mathrm{NC}$. Using the DSM, one can separate these spectra and establish their parameters.

\section{Results and discussion}

The results of applying the fourth derivative to the smoothed EPR spectrum of $\mathrm{ZnO}$ : Mn NC with a manganese content of 4 at. \% obtained by the cryochemical method at annealing temperature $\mathrm{T}=350{ }^{\circ} \mathrm{C}$ are shown in Fig. 1. The initial EPR spectrum of $\mathrm{ZnO}: \mathrm{Mn} \mathrm{NC}$ was smoothed by the Tikhonov method with a regularization coefficient $\alpha=5 \cdot 10^{6}$. Smoothing of all derivatives of the EPR spectrum of $\mathrm{ZnO}$ : Mn NC was carried out by the Tikhonov method with a regularization coefficient $\alpha=1 \cdot 10^{7}$. Such smoothing constants were used in connection with the actual smoothness of the initial spectrum. Fig. $1 \mathrm{~b}$ shows the second derivative of the EPR spectrum of $\mathrm{ZnO}$ : Mn NC. The analysis of the components of the EPR spectrum with respect to the second derivative of the spectrum is very difficult. This is due to the fact that in this derivative there are extrema located on one side of the abscissa axis. To identify a detailed picture, it is necessary to increase the order of the derivative.

In figure Fig.1c shows the fourth derivative of the EPR spectrum of the $\mathrm{ZnO}: \mathrm{Mn}$ NC. In areas with a magnetic field value of 0-240 $\mathrm{mT}$ and 380-500 mT, a background noise signal appeared. Subsequently, we analyzed the central information component of the EPR spectrum in the range of the magnetic field values of $240-380 \mathrm{mT}$. This part of the spectrum on an enlarged scale is shown in Fig. 1d. Such processing of the 
experimental spectrum by the DSM makes it possible to identify six intense HFS lines of the $\mathrm{Mn}^{2+}$ ions (transition $1 / 2 \rightarrow-1 / 2$ ) in the central part of the EPR spectrum, as well as six less intense lines on the right and left sides from the central part of the spectrum related to the HFS $\mathrm{Mn}^{2+}$ ions (transitions $1 / 2 \rightarrow 3 / 2$ and $-1 / 2 \rightarrow-3 / 2$ ). Such a spectrum is characterized by the HFS constant $A=76.53$ oersted and $g \approx 2.1$. Such a value of the $\mathrm{g}$ factor was observed in the EPR spectra of $\mathrm{ZnO} \mathrm{NCs}$ upon doping with $\mathrm{Mn}^{2+}$ ions under the simultaneous action of hydrogen [9].
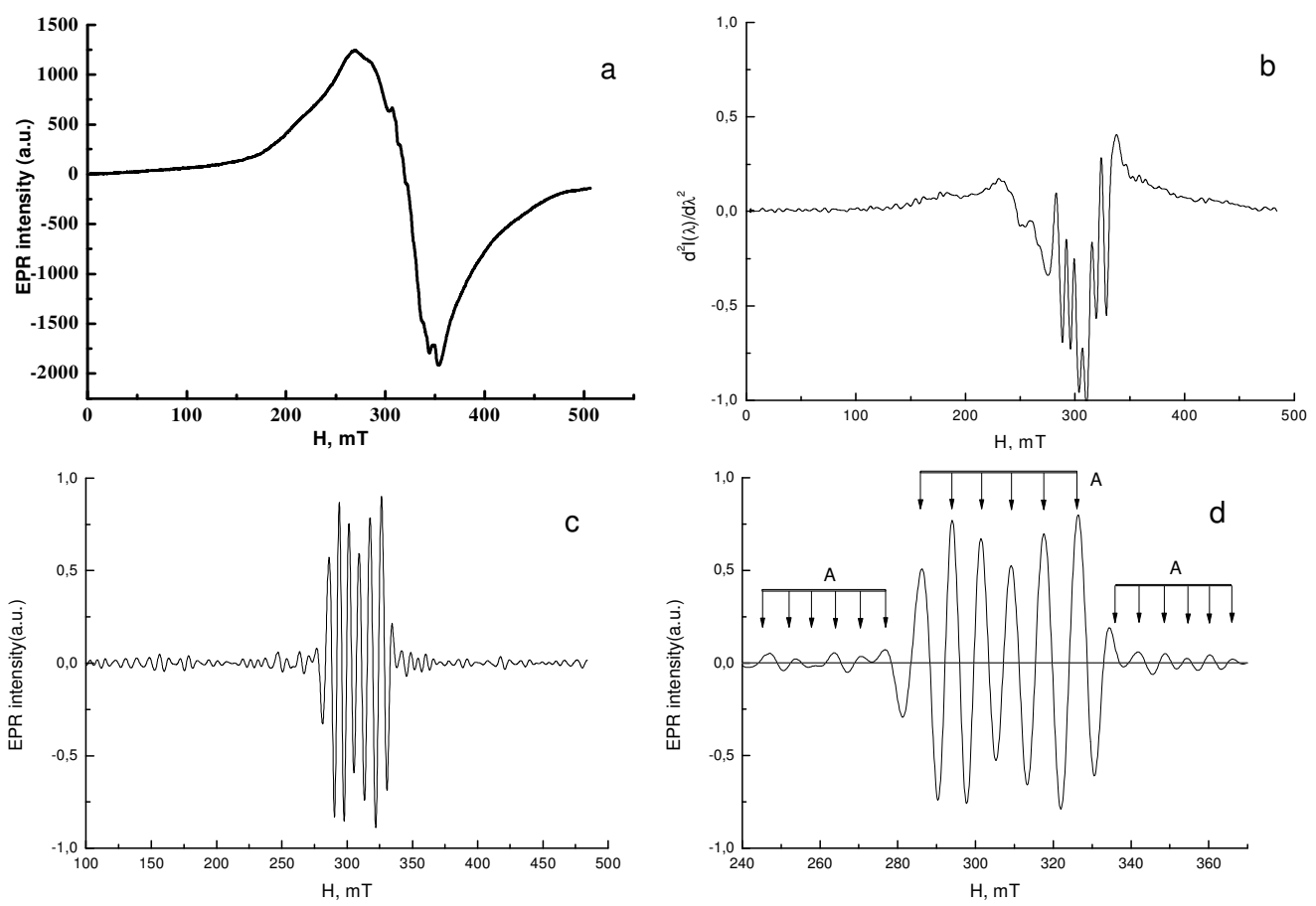

Fig. 1. Experimental EPR spectrum (a), second derivative (b), fourth derivative (c), extended central part of the spectrum of the fourth derivative (d) of the experimental spectrum of $\mathrm{ZnO}$ : Mn nanocrystals with a manganese content of 4 at. $\%$ obtained by the cryochemical method at annealing temperature $\mathrm{T}=350{ }^{\circ} \mathrm{C}$.
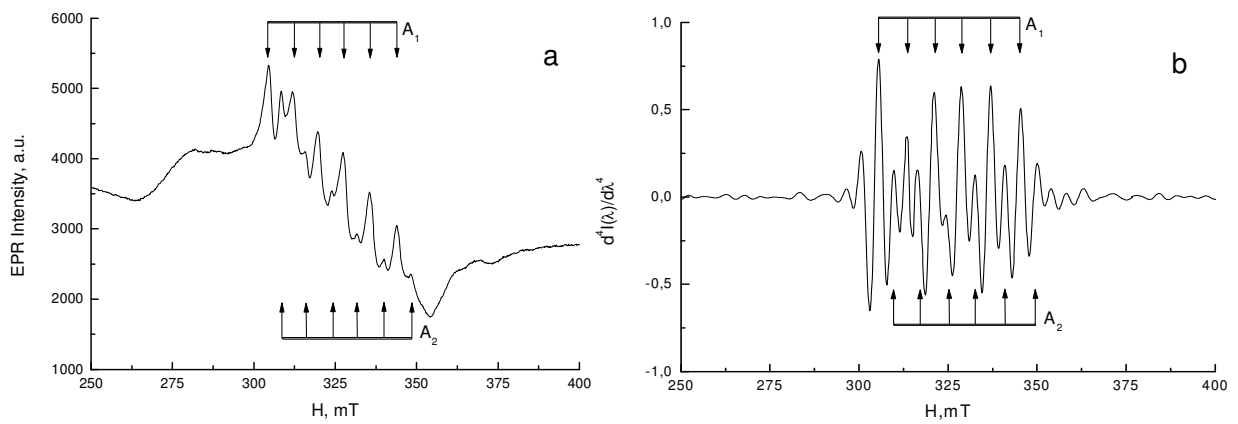

Fig. 2. The experimental EPR spectrum (a) and the fourth derivative of the experimental spectrum (b) of ZnO: Mn nanocrystals obtained by ultrasonic pyrolysis of an aerosol with a manganese content of 2 at. \% after annealing at a temperature of $T=550{ }^{\circ} \mathrm{C}$.

In figure Fig.2a shows the EPR spectrum of $\mathrm{ZnO}: \mathrm{Mn} \mathrm{NC}$ with an activator content of 2 at. \% obtained by the ultrasonic aerosol pyrolysis method after annealing at a temperature of $\mathrm{T}=550{ }^{\circ} \mathrm{C}$. It can be assumed that this spectrum is a superposition of the 
HFS of two EPR spectra of $\mathrm{Mn}^{2+}$ ions with the parameters of HFS $\mathrm{A}_{1}$ and $\mathrm{A}_{2}$ [3]. It is very difficult to precisely determine these constants. The results of applying the DSM based on the analysis of the fourth derivative with respect to this EPR spectrum allow this to be done. The initial EPR spectrum of $\mathrm{ZnO}$ : Mn nanocrystals was smoothed out using the Tikhonov method with a regularization coefficient $\alpha=7.6 \cdot 10^{3}$. The lower value of the regularization coefficient compared to the previous task is due to the smaller range of analysis of the EPR spectrum by the magnitude of the magnetic field, as well as highquality initial experimental data.

Smoothing of all derivatives of the EPR spectrum of $\mathrm{ZnO}$ : Mn NC was carried out by the Tikhonov method with a regularization coefficient $\alpha=1 \cdot 10^{7}$. In Fig. 2b two groups of HFS lines of $\mathrm{Mn}^{2+}$ ions with close constants $A_{1}=78.59$ and $A_{2}=80.45$ oerst are clearly visible and g-factors $g_{1} \approx 1.96$ and $g_{2} \approx 2.00$, respectively. According to published data, the spectrum is caused by $\mathrm{Mn}^{2+}$ ions that isovalently replace $\mathrm{Zn}^{2+}$ ions at the sites of the crystal lattice of $\mathrm{ZnO}$ nanocrystals, and the spectrum is associated with $\mathrm{Mn}^{2+}$ ions located in the deformed surface zone [4].

\section{Conclusions}

The application of DSM to the experimental EPR spectra made it possible to reveal the narrow EPR lines that make up the broad line of spectrum, determine the nature of the paramagnetic center of the broad line and the spectrum constant. The application of this method also made it possible to separate the EPR spectra superimposed on each other and determine the spectral constants. The results obtained indicate the prospects for the use of DSM in the analysis of complex experimental EPR spectra.

\section{References}

1. Bender, C. EPR: instrumental methods (Vol. 21) /Bender, C. and Berliner, L. 1st edition - Springer Science \& Business Media, 2004. - 328p.

2. Stoll, S., EasySpin, a comprehensive software package for spectral simulation and analysis in EPR / S. Stoll, A. Schweiger// Journal of magnetic resonance. - 2006. - Vol. 178. -P. 42-55.

3. Kovalenko, O.V. Photoluminescence and EPR spectrum of $\mathrm{ZnO}: \mathrm{Mn}$ nanocrystals / O.V. Kovalenko, M.F. Bulaniy, V.Y. Vorovskiy,O.V. Khmelenko// Journal of Physics and Electronics. - 2018. - Vol.26. - P.69-72.

4. Price, Yü. B. Effects of $\mathrm{MnO}$ doping on the electronic properties of zinc oxide: $406 \mathrm{GHz}$ electron paramagnetic resonance spectroscopy and Newman superposition model analysis / Yü.B. Price, G. Hardal, M. Açikgöz ,S. Repp, E. Erdem// Journal of Applied Physics. - 2015. -Vol.118. - P. 175705.

5. Kovalenko, A. V. Application of derivative spectroscopy method to photoluminescence in ZnS: Mn nanocrystals / A.V. Kovalenko, E.G. Plakhtiy, S.M. Vovk // Ukrainian Journal of Physical Optics. - 2018. - Vol. 19. - P. 133-140.

6. Kovalenko, O. V. Method of smoothing photoluminescence spectra / O.V. Kovalenko, S.M. Vovk, Ye.G. Plakhtii // Journal of Physics and Electronics. - 2018. - Vol. 26. - P. 73-80.

7. Kovalenko, O.V. Modification of Surface of ZnO: Mn Nanocrystals Synthesized by the Cryochemical Method / O.V. Kovalenko, V.Y. Vorovsky, M.F. Bulanyi, O.V. Khmelenko // Journal of Nano-and Electronic Physics. - 2019. - Vol.11. - P.05034

8. Bulaniy, M.F. Synthesis of $\mathrm{ZnO}$ and $\mathrm{ZnO}$ : Mn Nanopowders by Ultrasonic Spray Pyrolysis / M.F. Bulaniy, V.Y. Vorovsky, A.V. Kovalenko, O.V. Khmelenko// Journal of Nano-and Electronic Physics. - 2016. - Vol. 8 - P.2043.

9. Guskos, N. Magnetic Resonance Study of MnO/ZnO Nanopowders/ N. Guskos, G. Zolnierkiewicz, J. Typek, D. Sibera, U. Narkiewicz, W. Lojkowski// Acta Physica Polonica, A. - 2011. - Vol. 120. -P.1074-1079. 